\title{
On the non-Aggregation of Normal Adult Hemoglobin and the Aggregation of Sickle Cell Hemoglobin
}

\author{
Supporting Information
}

\author{
N. Galamba ${ }^{\mathrm{a}, *}$

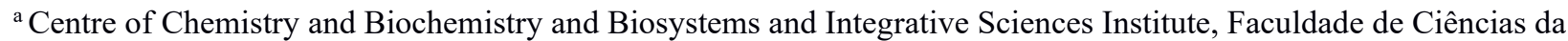 \\ Universidade de Lisboa, Edifício C8, Campo Grande, 1749-016 Lisboa, Portugal
}

\footnotetext{
*Corresponding author. Electronic mail: njgalamba@fc.ul.pt
} 


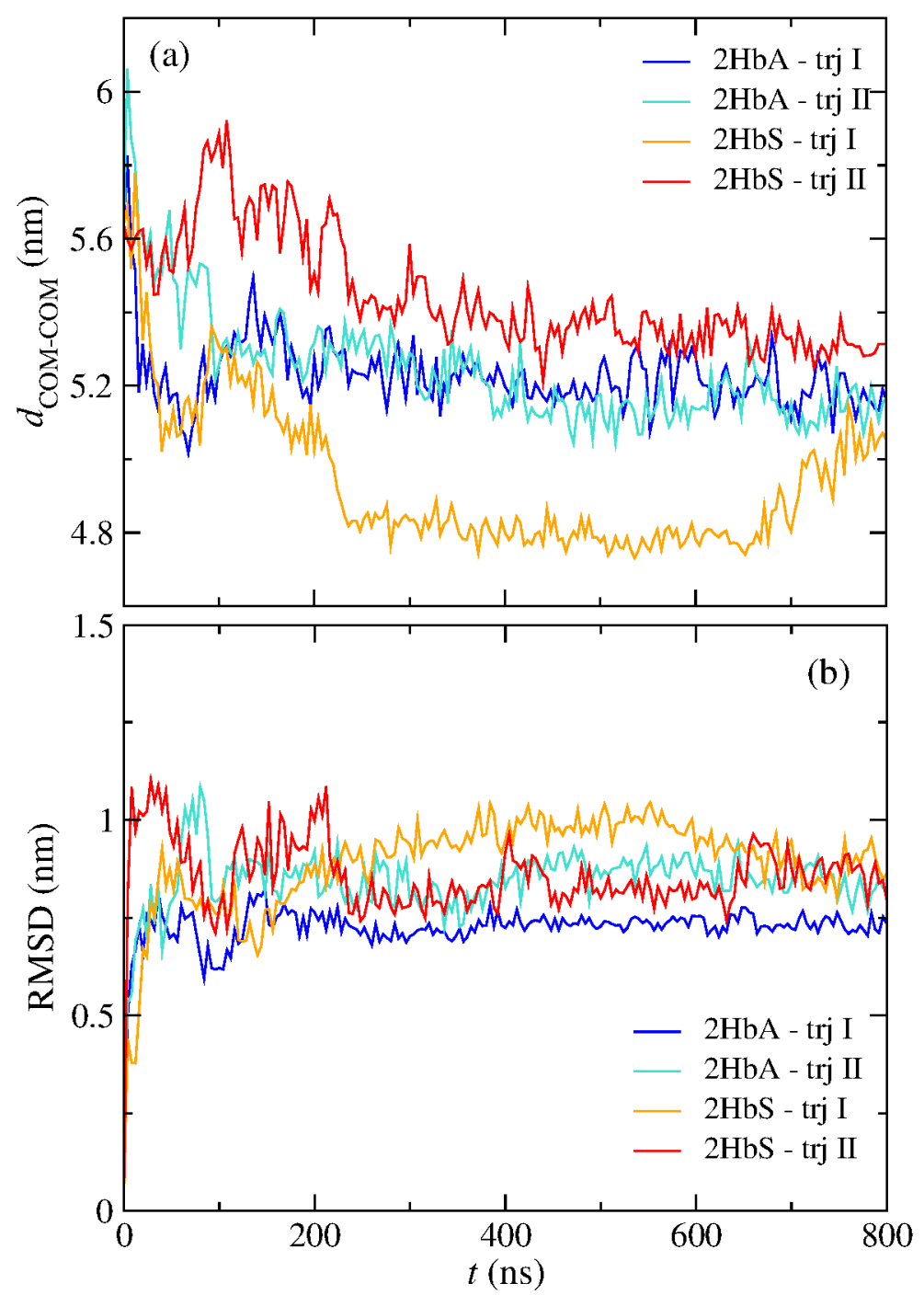

Figure 1-SI - (a) Centre of mass-centre of mass (COM-COM) distance between the $\mathrm{HbA}$ and the $\mathrm{HbS}$ tetramers during the $800 \mathrm{~ns}$ time-frame of the unrestrained simulations for trajectories I and II. (b) The respective root mean square deviation (RMSD), relative to the crystal structure, calculated for every atom of $2 \mathrm{HbA}$ and $2 \mathrm{HbS}$.

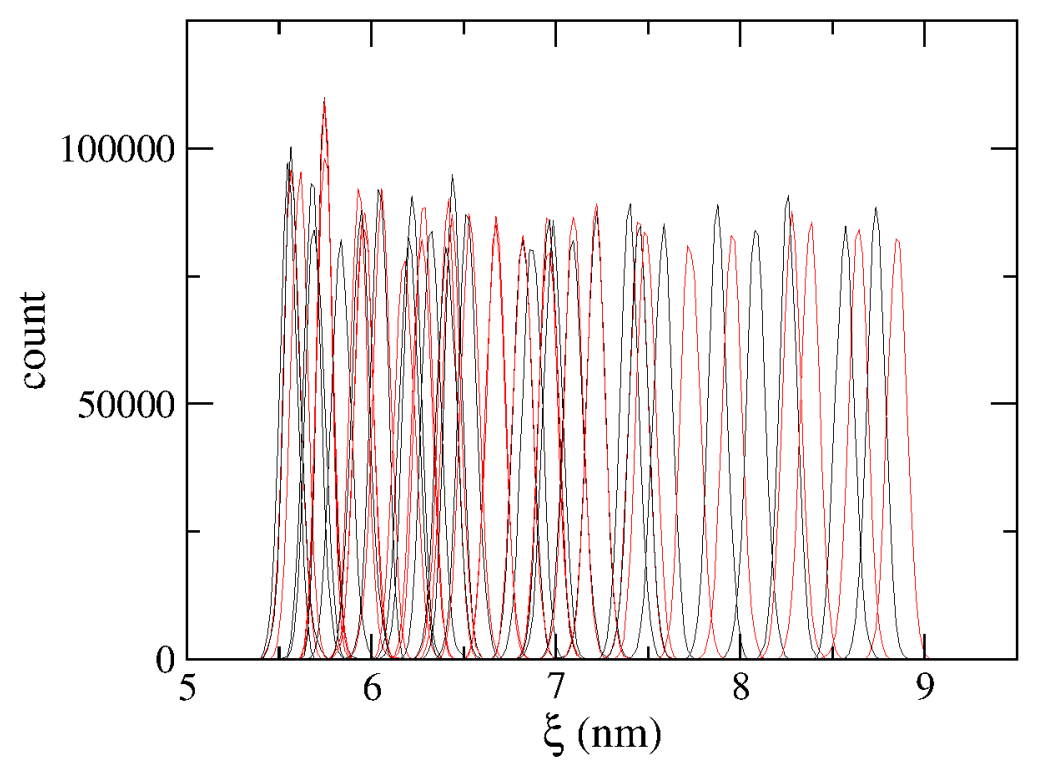

Figure 2-SI - Potential of mean force umbrella histograms for $2 \mathrm{HbA}$ at the different reaction coordinate distances. 
2HbA
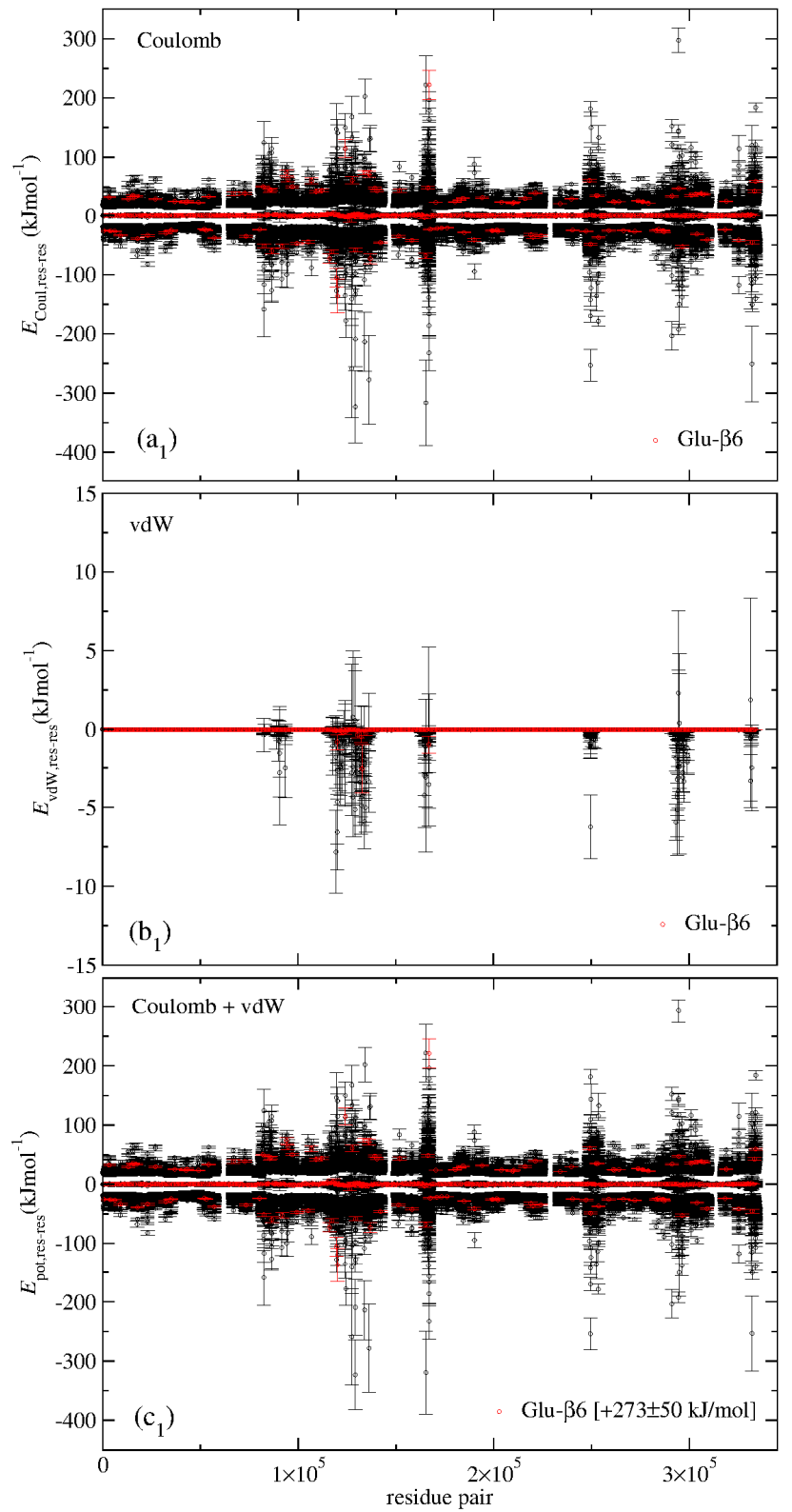

$2 \mathrm{HbS}$
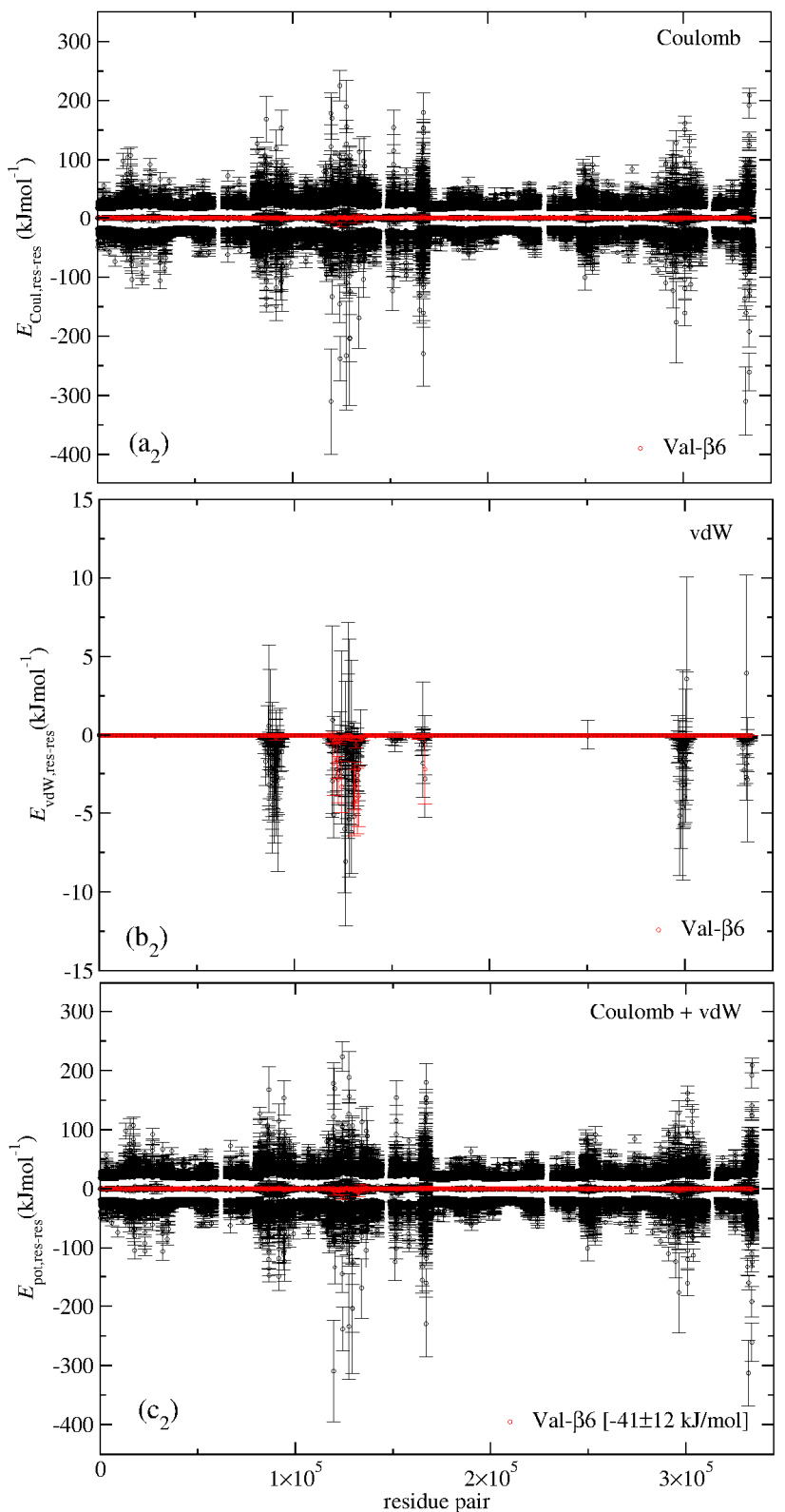

Figure 3-SI $-N^{2}$ intermolecular interactions for $2 \mathrm{HbS}$ and $2 \mathrm{HbA}$, where $N(N=578)$ is the number of residues and HEM groups of $\mathrm{HbS}$ and $\mathrm{HbA}$. (a) Coulombic, (b) van der Waals, (c) potential energy (electrostatic and van der Waals). Energies were calculated every $1 \mathrm{~ns}$ over the last $400 \mathrm{~ns}$ of the MD trajectories; the respective standard deviations are shown. The red points are interactions involving Glu- $\beta 6$ and Val- $\beta 6$ in $2 \mathrm{HbA}$ and $2 \mathrm{HbS}$, respectively. 


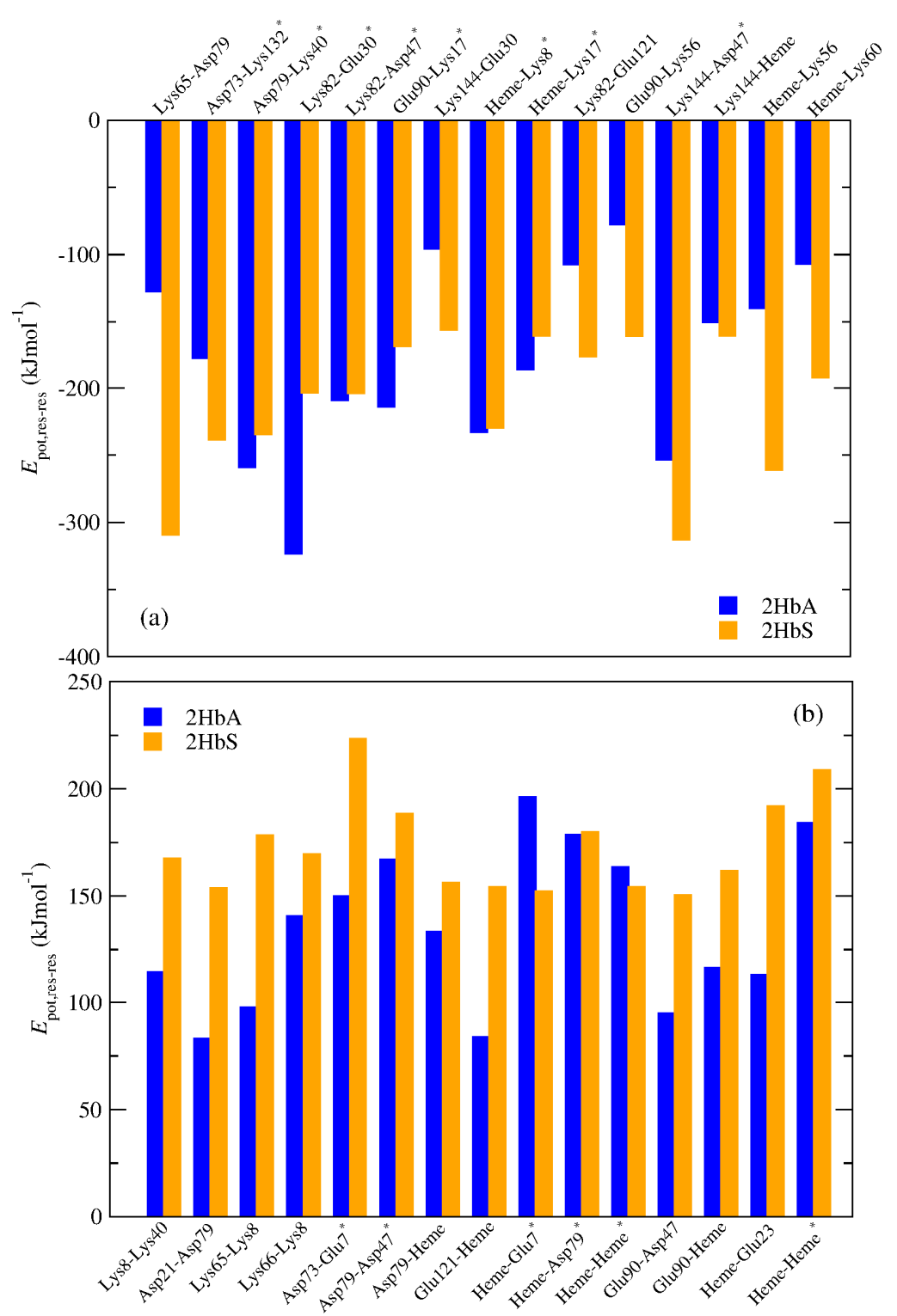

Figure 4-SI - Lowest and highest residue pair interactions in $2 \mathrm{HbS}$ and the respective energies for $2 \mathrm{HbA}$. The asterisk above some residue pairs indicates residue pairs also found among the lowest and highest energy interactions in 2HbA. Standard deviations for $2 \mathrm{HbS}$ are given in Tables 3-SI and 4-SI. 


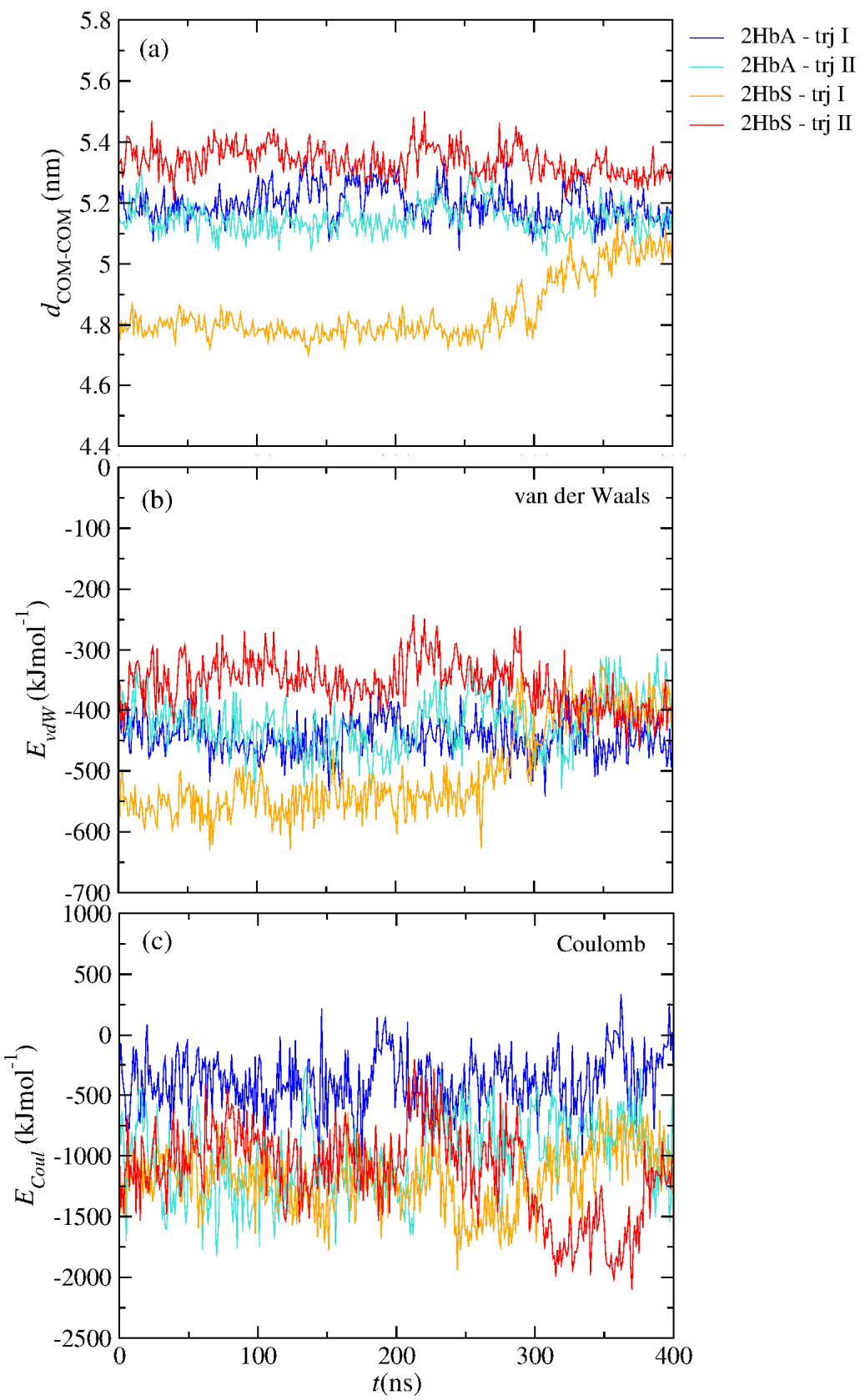

Figure 5-SI - (a) COM-COM distance (b) protein-protein van der Waals energy and (c) protein-protein electrostatic energy, for $2 \mathrm{HbA}$ and $2 \mathrm{HbS}$ calculated for the last $400 \mathrm{~ns}$ of trajectories I and II. 
Table 1-SI - 2HbA lowest mean potential energy residue pairs (salt bridges).

\begin{tabular}{|c|c|c|c|c|}
\hline \multicolumn{2}{|c|}{$\mathrm{HbA}-1$} & \multicolumn{2}{|c|}{$\mathrm{HbA}-2$} & \multirow{2}{*}{$\begin{array}{c}E_{p o t} \\
\left(\mathrm{kJmol}^{-1}\right)\end{array}$} \\
\hline Chain & Residue & Chain & Residue & \\
\hline $1 \beta_{1}$ & Asp 73 & $2 \beta_{2}$ & Lys132 & $-178 \pm 29$ \\
\hline $1 \beta_{1}$ & Asp79 & $2 \alpha_{2}$ & Lys40 & $-259 \pm 82$ \\
\hline $1 \beta_{1}$ & Lys82 & $2 \alpha_{2}$ & Glu30 & $-323 \pm 59$ \\
\hline $1 \beta_{1}$ & Lys 82 & $2 \alpha_{2}$ & Asp47 & $-209 \pm 47$ \\
\hline $1 \beta_{1}$ & Glu90 & $2 \beta_{2}$ & Lys17 & $-214 \pm 50$ \\
\hline $1 \beta_{1}$ & Asp94 & $2 \beta_{2}$ & Lys17 & $-278 \pm 74$ \\
\hline $1 \beta_{1}$ & Lys144 & $2 \beta_{2}$ & Glu121 & $-319 \pm 70$ \\
\hline $1 \beta_{1}$ & Heme & $2 \beta_{2}$ & Lys8 & $-233 \pm 30$ \\
\hline $1 \beta_{1}$ & Heme & $2 \beta_{2}$ & Lys17 & $-186 \pm 21$ \\
\hline $1 \beta_{2}$ & N-term Val1 & $2 \alpha_{2}$ & Glu23 & $-170 \pm 11$ \\
\hline $1 \beta_{2}$ & N-term Vall & $2 \alpha_{2}$ & Glu127 & $-254 \pm 27$ \\
\hline $1 \beta_{2}$ & Lys8 & $2 \alpha_{2}$ & Glu23 & $-179 \pm 8$ \\
\hline $1 \beta_{2}$ & Asp73 & $2 \alpha_{2}$ & Lys56 & $-204 \pm 24$ \\
\hline $1 \beta_{2}$ & Asp79 & $2 \alpha_{2}$ & Arg31 & $-192 \pm 9$ \\
\hline $1 \beta_{2}$ & Lys144 & $2 \alpha_{2}$ & Asp47 & $-253 \pm 63$ \\
\hline
\end{tabular}

Table 2-SI - 2HbA highest mean potential energy residue pairs (repulsions).

\begin{tabular}{|c|c|c|c|c|}
\hline \multicolumn{2}{|c|}{$\mathrm{HbA}-1$} & \multicolumn{2}{|c|}{$\mathrm{HbA}-2$} & \multirow{2}{*}{$\begin{array}{c}E_{p o t} \\
\left(\mathrm{kJmol}^{-1}\right)\end{array}$} \\
\hline Chain & Residue & Chain & Residue & \\
\hline $1 \beta 1$ & Lys65 & $2 \beta 2$ & N-term Vall & $146 \pm 42$ \\
\hline $1 \beta_{1}$ & Asp73 & $2 \beta 2$ & Glu7 & $150 \pm 23$ \\
\hline $1 \beta_{1}$ & Asp79 & $2 \alpha_{2}$ & Asp47 & $167 \pm 34$ \\
\hline $1 \beta_{1}$ & Glu90 & $2 \beta_{2}$ & Glu121 & $202 \pm 29$ \\
\hline $1 \beta_{1}$ & Lys144 & $2 \beta_{2}$ & Lys17 & $222 \pm 49$ \\
\hline $1 \beta_{1}$ & Heme & $2 \beta_{2}$ & Glu6 & $221 \pm 25$ \\
\hline $1 \beta_{1}$ & Heme & $2 \beta_{2}$ & Glu7 & $196 \pm 14$ \\
\hline $1 \beta_{1}$ & Heme & $2 \beta_{2}$ & Asp73 & $145 \pm 7$ \\
\hline $1 \beta_{1}$ & Heme & $2 \beta_{2}$ & Asp79 & $179 \pm 16$ \\
\hline $1 \beta_{1}$ & Heme & $2 \beta_{2}$ & Heme & $164 \pm 4$ \\
\hline $1 \beta_{2}$ & N-term Vall & $2 \alpha_{2}$ & Arg31 & $182 \pm 12$ \\
\hline $1 \beta_{2}$ & Asp73 & $2 \alpha_{2}$ & Glu23 & $152 \pm 11$ \\
\hline $1 \beta_{2}$ & Asp79 & $2 \alpha_{2}$ & Glu27 & $294 \pm 20$ \\
\hline $1 \beta_{2}$ & Asp79 & $2 \alpha_{2}$ & Glu30 & $145 \pm 10$ \\
\hline $1 \beta_{2}$ & Heme & $2 \alpha_{2}$ & Heme & $184 \pm 8$ \\
\hline
\end{tabular}


Table 3-SI - 2HbS lowest mean potential energy residue pairs (salt bridges).

\begin{tabular}{|c|c|c|c|c|}
\hline \multicolumn{2}{|c|}{ HbS-1 } & \multicolumn{2}{|c|}{ HbS-2 } & \multirow{2}{*}{$\begin{array}{c}E_{p o t} \\
\left(\mathrm{kJmol}^{-1}\right)\end{array}$} \\
\hline Chain & Residue & Chain & Residue & \\
\hline $1 \beta_{1}$ & Lys65 & $2 \beta_{2}$ & Asp79 & $-310 \pm 86$ \\
\hline $1 \beta_{1}$ & Asp73 & $2 \beta_{2}$ & Lys132 & $-239 \pm 37$ \\
\hline $1 \beta_{1}$ & Asp79 & $2 \alpha_{2}$ & Lys40 & $-234 \pm 89$ \\
\hline $1 \beta_{1}$ & Lys 82 & $2 \alpha_{2}$ & Glu30 & $-204 \pm 110$ \\
\hline $1 \beta_{1}$ & Lys82 & $2 \alpha_{2}$ & Asp47 & $-204 \pm 40$ \\
\hline $1 \beta_{1}$ & Glu90 & $2 \beta_{2}$ & Lys17 & $-169 \pm 51$ \\
\hline $1 \beta_{1}$ & Lys 144 & $2 \alpha_{2}$ & Glu30 & $-156 \pm 22$ \\
\hline $1 \beta_{1}$ & Heme & $2 \beta_{2}$ & Lys8 & $-230 \pm 56$ \\
\hline $1 \beta_{1}$ & Heme & $2 \beta_{2}$ & Lys17 & $-161 \pm 24$ \\
\hline $1 \beta_{2}$ & Lys82 & $2 \beta_{2}$ & Glu121 & $-177 \pm 69$ \\
\hline $1 \beta_{2}$ & Glu90 & $2 \alpha_{2}$ & Lys56 & $-161 \pm 22$ \\
\hline $1 \beta_{2}$ & Lys144 & $2 \alpha_{2}$ & Asp47 & $-313 \pm 56$ \\
\hline $1 \beta_{2}$ & Lys 144 & $2 \alpha_{2}$ & Heme & $-161 \pm 12$ \\
\hline $1 \beta_{2}$ & Heme & $2 \alpha_{2}$ & Lys56 & $-261 \pm 32$ \\
\hline $1 \beta_{2}$ & Heme & $2 \alpha_{2}$ & Lys60 & $-192 \pm 29$ \\
\hline
\end{tabular}

Table 4-SI - 2HbS highest mean potential energy residue pairs (repulsions) .

\begin{tabular}{ccccc}
\hline \multicolumn{2}{c}{ HbS-1 } & \multicolumn{2}{c}{ HbS-2 } & $\begin{array}{c}E_{\text {pot }} \\
\left(\mathrm{kJmol}^{-1}\right)\end{array}$ \\
\hline $1 \beta_{1}$ & Lys8 & $2 \alpha_{2}$ & Lys40 & $168 \pm 38$ \\
$1 \beta_{1}$ & Asp21 & $2 \beta_{2}$ & Asp79 & $154 \pm 30$ \\
$1 \beta_{1}$ & Lys65 & $2 \beta_{2}$ & Lys8 & $178 \pm 35$ \\
$1 \beta_{1}$ & Lys66 & $2 \beta_{2}$ & Lys8 & $170 \pm 35$ \\
$1 \beta_{1}$ & Asp73 & $2 \beta_{2}$ & Glu7 & $223 \pm 25$ \\
$1 \beta_{1}$ & Asp79 & $2 \alpha_{2}$ & Asp47 & $189 \pm 44$ \\
$1 \beta_{1}$ & Asp79 & $2 \alpha_{2}$ & Heme & $156 \pm 11$ \\
$1 \beta_{1}$ & Glu121 & $2 \beta_{1}$ & Heme & $154 \pm 29$ \\
$1 \beta_{1}$ & Heme & $2 \beta_{2}$ & Glu7 & $152 \pm 16$ \\
$1 \beta_{1}$ & Heme & $2 \beta_{2}$ & Asp79 & $180 \pm 32$ \\
$1 \beta_{1}$ & Heme & $2 \beta_{2}$ & Heme & $154 \pm 9$ \\
$1 \beta_{2}$ & Glu90 & $2 \alpha_{2}$ & Asp47 & $151 \pm 11$ \\
$1 \beta_{2}$ & Glu90 & $2 \alpha_{2}$ & Heme & $162 \pm 12$ \\
$1 \beta_{2}$ & Heme & $2 \alpha_{2}$ & Glu23 & $192 \pm 21$ \\
$1 \beta_{2}$ & Heme & $2 \alpha_{2}$ & Heme & $209 \pm 12$ \\
\hline
\end{tabular}

